\title{
RESEARCH
}

\section{Regional opening strategies with commuter testing and containment of new SARS-CoV-2 variants}

\author{
Martin J. Kühn ${ }^{1 *}$, Daniel Abele ${ }^{1}$, Sebastian Binder ${ }^{2 *}$, Kathrin Rack ${ }^{1}$, Margrit Klitz ${ }^{1}$, Jan Kleinert ${ }^{1}$, Jonas \\ Gilg$^{1}$, Luca Spataro ${ }^{1}$, Wadim Koslow ${ }^{1}$, Martin Siggel ${ }^{1}$, Michael Meyer-Hermann ${ }^{2 *}$ and Achim \\ Basermann ${ }^{1 *}$
}

\footnotetext{
${ }^{*}$ Correspondence:

Martin.Kuehn@DLR.de;

SB@Theoretical-Biology.de; MMH@Theoretical-Biology.de;

Achim.Basermann@DLR.de

${ }^{1}$ Institute for Software Technology,

German Aerospace Center,

Cologne, Germany

${ }^{2}$ Department of Systems

Immunology and Braunschweig Integrated Centre of Systems Biology (BRICS), Helmholtz Centre for Infection Research, Braunschweig, Germany

Full list of author information is available at the end of the article
}

\begin{abstract}
Background: Despite the accelerating vaccination process, a large majority of the population is still susceptible to SARS-CoV-2. In addition, we face the spread of novel variants. Until we overcome the pandemic, reasonable mitigation and opening strategies are crucial to balance public health and economic interests.
\end{abstract}

Methods: We model the spread of SARS-CoV-2 over the German counties by a graph-SIR-type model with particular focus on commuter testing. We account for political interventions by varying contact reduction values in private and public locations such as homes, schools, workplaces, and other. We consider different levels of lockdown strictness, commuter testing strategies, or the delay of intervention implementation. We conduct numerical simulations to assess the effectiveness of the different intervention strategies after one month. The virus dynamics in the counties are initialized randomly with incidences between 75-150 weekly new cases per 100,000 inhabitants (red zones) or below 10 (green zones) and consider 25 different initial scenarios of randomly distributed red zones (between 2 and $20 \%$ of all counties). To account for uncertainty, we consider an ensemble set of 500 Monte Carlo runs for each scenario.

Results: We find that the strength of the lockdown in regions with out of control virus dynamics is most important to avoid the spread into neighboring regions. With very strict lockdowns in red zones, commuter testing rates of twice a week can substantially contribute to the safety of adjacent regions. In contrast, less strict lockdowns with the same commuter testing rate quickly and substantially lead to overall higher infection dynamics. A further key contributor is the potential delay of the intervention implementation. In order to keep the spread of the virus under control, strict regional lockdowns with minimum delay and commuter testing of at least twice a week are advisable.

Conclusions: Our results indicate that local containment of outbreaks and maintenance of low overall incidence is possible even in densely populated and highly connected areas. While we demonstrate this on data from Germany, similar patterns of mobility likely exist in many countries and our results are, hence, generalizable to a certain extent.

Keywords: SARS-CoV-2; Covid-19; Nonpharmaceutical intervention; Mitigation strategy; Modeling; Predictive Analytics; NoCovid strategy 


\section{Background}

With about three million detected infections in Germany [1] and about 22 million vaccinations [2] we can reasonably assume that at least $80 \%$ of the population are still susceptible to SARS-CoV-2. New SARS-CoV-2 variants (B.1.1.7, B.1.351 and P.1) contribute to the recent increase in case numbers [3, Report of Apr. 7]. Due to the higher transmission risk of B.1.1.7 [4], previous mitigation strategies have to be reconsidered and strengthened.

The prediction of SARS-CoV-2 infections by mathematical models is an active area of research of many groups from all over the world. In particular for Germany, e.g., the authors of $[5,6,7,8,9,10,11,12]$ have studied the effects of political interventions and provided predictions for the development of new cases. Spatial resolutions with a particular focus on networks and mobility was, among others, presented by the authors of $[5,9,12,11]$.

A proactive approach to fight SARS-CoV-2 in Germany and Europe is presented in [13] with the aim of a safe and sustainable re-opening of societies and economies; see also related discussions in $[14,15,16]$. Lockdowns remain implemented regionally until the incidence is below 10 cases per week and 100,000 inhabitants and local measures are reintroduced rapidly should infections flare up again. This approach was already successfully implemented in Australia. It is based on the observation that the virus spreads heterogeneously: There are certain regions with very low incidences while other regions ("hotspots") are highly affected [3]. However, neighboring regions can quickly become impacted, especially, by daily commuting. So far, the feasibility and effectiveness of the strategy with respect to commuter testing and local lockdowns has not been investigated numerically. The aim of this paper is a quantification of the necessary test frequency, the required strength of the local lockdown and the time frame that we have for the intervention implementation to avoid the spreading of the virus to neighboring regions.

\section{Methods}

The aim of this study is to provide viable strategies of careful opening of facilities in low-incidence regions without being affected by neighboring regions of substantially higher incidence. Motivated by [13], the regions (here: German counties) are partitioned into red and green zones. A region is labeled a green zone if there is a stable low incidence below 12 per week and 100,000 inhabitants ${ }^{[1]}$ with effective tracing of new cases. As soon as this is no longer the case, a region is labeled a red zone.

We initialize the set of German counties randomly with weekly incidences per 100,000 inhabitants of around 5 for green zones and 75-150 for red zones. We consider 25 different random scenarios with in between 2 and $20 \%$ of the counties as red zones; cf. Fig. 3 (top). In green zones, all facilities are open with only protective measures, such as distancing and face masks, in place. Red zones start from an incidence of 12, and in that case a lockdown is implemented for 30 days. For counties with incidence 100 or higher enforced measures are applied. Since political decisions require time, intervention implementation allows for certain delay. Commuter

[1] The small deviation from the incidence 10 in [13] takes into account reporting delays. 


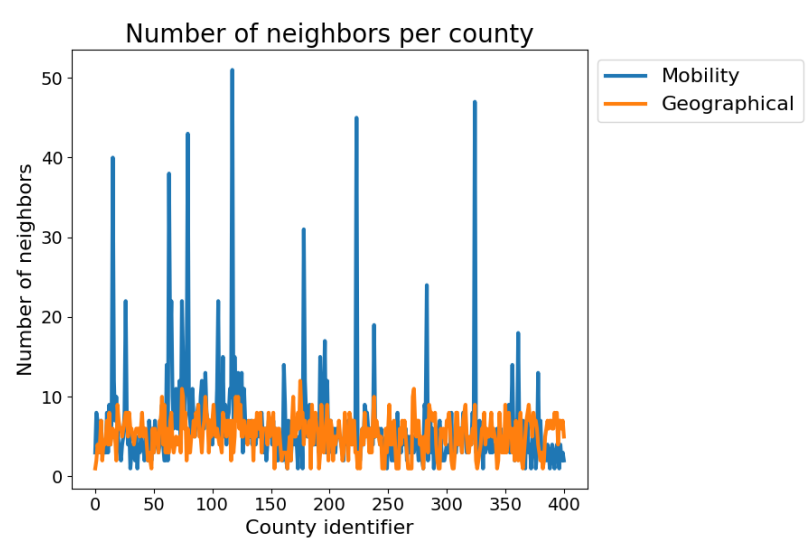

Figure 1 Number of neighboring zones according to geographical and mobility definitions.

testing is conducted for all commuters coming from red zones. If tested positive, presymptomatic, asymptomatic or symptomatic people will be isolated and prevented from traveling or commuting before recovery. For the precise set of values for lockdowns or testing rates, see the section on mitigation and opening strategies. To assess the impact of the initial overall incidence, we also present two scenarios with $42 \%$ and $60 \%$ of counties classified red at the beginning.

Note that throughout this work, incidence always refers to the incidence averaged over 100000 people and 7 days.

\section{Mathematical model}

Our mathematical model for the spread of SARS-CoV-2 accounts for agedependence [17, 18, 19], heterogeneous spread across regions [3], and commuter testing for mitigation [20].

For the geographic resolution, we use a graph approach as presented in [11] and assign one SIR-type model to each German county. The resulting models will be coupled by the edges of the graph which represent the mobility between the regions. The mobility data is obtained from the German Federal Employment Agency [21] complemented with geo-referenced Twitter data [22]. For more details on the data and the practical exchange between regions, we refer to [11]. In the graph, counties are not only connected to their geographical neighbors. We define neighbors by mobility, depending on the numbers of in-commuters. If the number of daily incommuters in county A (coming from county B) exceeds 1000 on average, B will be classified a neighbor of $\mathrm{A}$. This leads to an increased number of neighbors for larger cities in particular as shown in Fig. 1.

For each county, we use an age-resolved SIR-type model based on $[8,11]$, with particular new focus on commuter

testing. The model uses the compartments Susceptible $\left(S_{i}\right)$; Exposed $\left(E_{i}\right)$, who carry the virus but are not yet infectious to others; Carriers $\left(C_{i}\right)$, who carry the virus and are infectious to others but do not yet show symptoms (they may be preor asymptomatic); Infected $\left(I_{i}\right)$, who carry the virus, are infectious and show symptoms; Hospitalized $\left(H_{i}\right)$, who experience a severe development of the disease; In 
Intensive Care Unit $\left(U_{i}\right)$; Dead $\left(D_{i}\right)$; and Recovered $\left(R_{i}\right)$, who cannot be infected again. To resolve age-specific disease parameters, the totality of people $N$ into $n$ different age groups $i=1, \ldots, n$. Note that our focus in this paper is not on hospitalizations, ICU bottlenecks, deaths or vaccinations such that these compartments are of minor interest for our considerations here.

In order to model commuter testing, we introduce the new compartments $C_{i}^{+}$ and $I_{i}^{+}$for carriers or infectious who are tested positive while commuting. The compartment $C_{i}^{+}$does not have any natural influx and only depends on the number of commuters and testing rates defined between counties on a daily basis. $I_{i}^{+}$has only influx from $C_{i}^{+}$and can also increase due to testing results. For a visualization of the process see Fig. 2. Testing within counties and lockdown strictness will be handled as presented in [11], adapted to counterstrategies against novel SARS-CoV2 variants. The full system of equations writes

$$
\begin{aligned}
\frac{d S_{i}}{d t} & =-S_{i} \rho_{i} \sum_{j=1}^{n} \phi_{i, j} \frac{\tilde{\beta}_{C, j} C_{j}+\tilde{\beta}_{I, j} I_{j}}{N_{j}}, \\
\frac{d E_{i}}{d t} & =S_{i} \rho_{i} \sum_{j=1}^{n} \phi_{i, j} \frac{\tilde{\beta}_{C, j} C_{j}+\tilde{\beta}_{I, j} I_{j}}{N_{j}}-\frac{1}{T_{E_{i}}^{C_{i}}} E_{i}, \\
\frac{d C_{i}}{d t} & =\frac{1}{T_{E_{i}}^{C_{i}}} E_{i}-\left(\frac{1-\mu_{C_{i}}^{R_{i}}}{T_{C_{i}}^{I_{i}}}+\frac{\mu_{C_{i}}^{R_{i}}}{T_{C_{i}}^{R_{i}}}\right) C_{i}, \\
\frac{d C_{i}^{+}}{d t} & =-\left(\frac{1-\mu_{C_{i}}^{R_{i}}}{T_{C_{i}}^{I_{i}}}+\frac{\mu_{C_{i}}^{R_{i}}}{T_{C_{i}}^{R_{i}}}\right) C_{i}^{+}, \\
\frac{d I_{i}}{d t} & =\frac{1-\mu_{C_{i}}^{R_{i}}}{T_{C_{i}}^{I_{i}}} C_{i}-\left(\frac{1-\mu_{I_{i}}^{H_{i}}}{T_{I_{i}}^{R_{i}}}+\frac{\mu_{I_{i}}^{H_{i}}}{T_{I_{i}}^{H_{i}}}\right) I_{i}, \\
\frac{d I_{i}^{+}}{d t} & =\frac{1-\mu_{C_{i}}^{R_{i}}}{T_{C_{i}}^{I_{i}}} C_{i}^{+}-\left(\frac{1-\mu_{I_{i}}^{H_{i}}}{T_{I_{i}}^{R_{i}}}+\frac{\mu_{I_{i}}^{H_{i}}}{T_{I_{i}}^{H_{i}}}\right) I_{i}^{+}, \\
\frac{d H_{i}}{d t} & =\frac{\mu_{I_{i}}^{H_{i}}}{T_{I_{i}}^{H_{i}}} I_{i}+\frac{\mu_{I_{i}}^{H_{i}}}{T_{I_{i}}^{H_{i}}} I_{i}^{+}-\left(\frac{1-\mu_{H_{i}}^{U_{i}}}{T_{H_{i}}^{R_{i}}}+\frac{\mu_{H_{i}}^{U_{i}}}{T_{H_{i}}^{U_{i}}}\right) H_{i}, \\
\frac{d U_{i}}{d t} & =\frac{\mu_{H_{i}}^{U_{i}}}{T_{H_{i}}^{U_{i}}} H_{i}-\left(\frac{1-\mu_{U_{i}}^{D_{i}}}{T_{U_{i}}^{R_{i}}}+\frac{\mu_{U_{i}}^{D_{i}}}{T_{U_{i}}^{D_{i}}}\right) U_{i}, \\
\frac{d R_{i}}{d t} & =\frac{\mu_{C_{i}}^{R_{i}}}{T_{C_{i}}^{R_{i}}}\left(C_{i}+C_{i}^{+}\right)+\frac{1-\mu_{I_{i}}^{H_{i}}}{T_{I_{i}}^{R_{i}}}\left(I_{i}+I_{i}^{+}\right)+\frac{1-\mu_{H_{i}}^{U_{i}}}{T_{H_{i}}^{R_{i}}} H_{i}+\frac{1-\mu_{U_{i}}^{D_{i}}}{T_{U_{i}}^{R_{i}}} U_{i}, \\
\frac{d D_{i}}{d t} & =\frac{\mu_{U_{i}}^{D_{i}}}{T_{U_{i}}^{D_{i}}} U_{i} .
\end{aligned}
$$

For each age group $i=1, \ldots, n$, we denote the transmission risk by $\rho_{i}$ and the proportion of carriers and infected people not isolated or quarantined is denoted by $\tilde{\beta}_{C, i}$ and $\tilde{\beta}_{I, i}$, respectively. The contact frequency matrix $\Phi=\left(\phi_{i, j}\right)_{i, j=1, \ldots, n}$ represents the (mean) daily contacts of a person of age group $i$ with people from age group $j$. For the remaining parameters, we use the variables $T_{*_{1}}^{*_{2}}$ for the time spent in state $*_{1} \in \mathcal{Z}_{i}$ before transition to state $*_{2} \in \mathcal{Z}_{i}$ and $\mu_{*_{1}}^{*_{2}}$ for the probability of a patient to go to state $*_{2}$ from state $*_{1}$. 


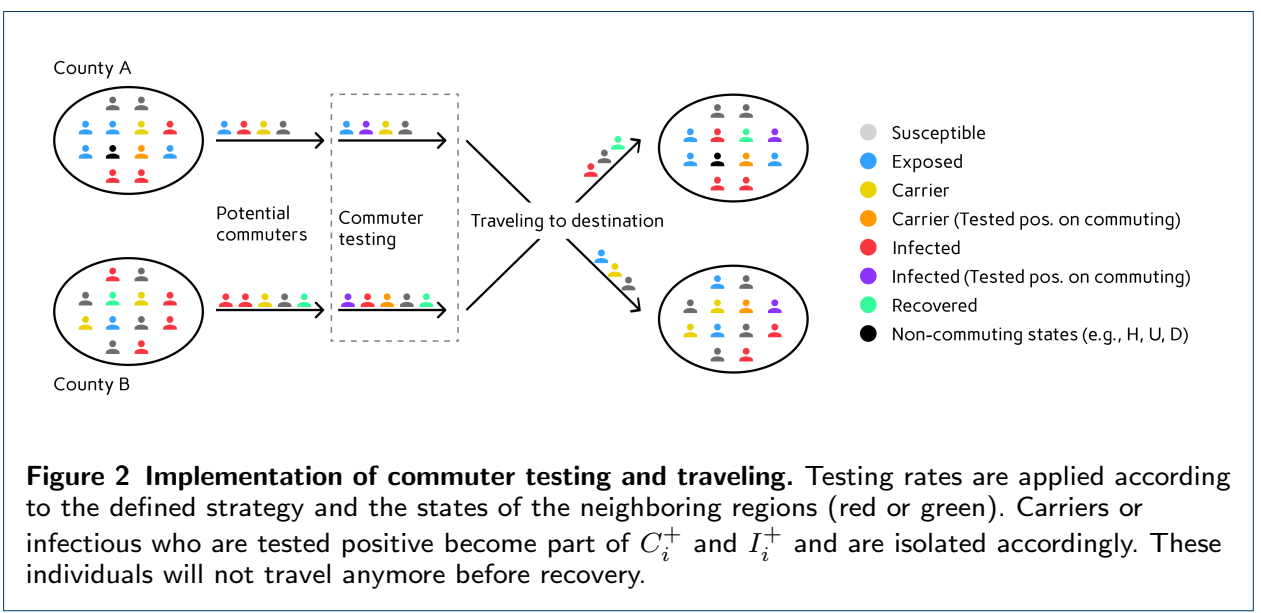

Except for the transmission risk $\rho_{i}$, the detection of carriers $\tilde{\beta}_{C, i}$ and the isolation and quarantine of infected $\tilde{\beta}_{I, i}$ defined in Table 1 , we use the parameter ranges and age groups as gathered and described elaborately in [11, Table 2]. To account for the variant B.1.1.7 in Germany [3, Report of Apr. 7], we use a 1.4 times increased value for the transmission risk $\rho_{i}$ [4]. The sigmoidal cosine curves in Table 1 are defined by the actual incidence of the zone. Thus, the minimum values are adopted for incidences below 10, where even carriers are quarantined and symptomatic are isolated fast and efficiently. From incidence 20 onward, carriers are generally no longer detected on a larger scale and the non-isolation of symptomatic increases to its highest value at incidence 150 .

The range of contact patterns depends on the decreed non-pharmaceutical interventions. The baseline number of contacts $\phi_{B, i, j}$ is obtained from $[23,24]$ as described in [11, Sec. 3.2]. The resulting number of contacts according to the nonpharmaceutical intervention then writes

$$
\phi_{i, j}=\sum_{* \in\{H, S, W, O\}}\left(1-\prod_{l=1}^{2} r_{*}^{(l)}\right) \phi_{M, *, i, j} .
$$

Here, $* \in\{H, S, W, O\}$ refers to the four locations of contact home, school, work, and other, and $r_{*}^{(l)} \in[0,1]$ is the reduction factor in effective contacts as induced from political decisions. The superindex $l$ is the intervention level. With $l=1$ we describe interventions that yield direct contact reduction such as gathering bans. With $l=2$ we include protective effects from, e.g., face masks and distancing; cf. [11] for more details. Precise values are provided in the following section. In contrast to [11], $r_{*}^{(l)}$ will not reduce the number of commuters from one region to another. The reduction in traveling of infectious and carriers will be based upon the isolation of symptomatic cases in the home region through $\widetilde{\beta}_{I_{i}}$ and the commuter testing rate as described in Fig. 2 and in the following section.

In order to account for the uncertainty, we consider an ensemble set of 500 Monte Carlo runs for each scenario such that our final results are based on 200000 different runs. 
range in age group

\begin{tabular}{|c|c|c|c|c|c|}
\hline param. & $0-4$ & $5-14$ & $15-34$ & $60-79$ & $80+$ \\
\hline$\rho^{(0)}$ & {$[0.028,0.056]$} & \multicolumn{2}{|c|}{$[0.070,0.098]$} & {$[0.11,0.14]$} & {$[0.21,0.28]$} \\
\hline$\tilde{\beta}_{C}$ & \multicolumn{5}{|c|}{ sigmoidal cosine curve from 0.5 to 1.0} \\
\hline$\tilde{\beta}_{I}$ & \multicolumn{5}{|c|}{ sigmoidal cosine curve from $[0.0,0.2]$ to $[0.4,0.5]$} \\
\hline
\end{tabular}

Table 1 Modified parameter ranges in comparison to [11]. We omit the age index $i$ for better readability.

\section{Mitigation and opening strategies}

Our tensor space of strategies summarized in Table 2 provides 16 different strategies. These are defined by the dimensions lockdown strictness for red zones, commuter testing rate from red zones, and delay of intervention implementation. A set of restrictions always lasts for 30 days and will only be lifted (red zones become green) if the incidence is below 12 at the end of the intervention.

For all strategies, the handling of green zones is identical. We assume that all facilities can be opened and that first level political interventions are unnecessary, i.e., $r_{*}^{(1)}=0$ with $* \in\{H, S, W, O\}$ in eq. (11). However, second level interventions with face masks and distancing are in place, and we assume a reduced contact rate by $r_{*}^{(2)} \in[0.25,0.35]$ for $* \in\{S, W, O\}$ (schools, workplaces and other), and $r_{H}^{(2)}=0$ (homes), since face masks may not be worn in most private situations if the spread is under control.

For red zones, we consider two different sets of lockdowns. Both differ in their severeness for incidences below 100 and above 100. For the stricter set denoted by $\mathbf{L}+$ and incidences below 100, we implement first level interventions $(l=1)$ that achieve an average contact reduction by $50 \%$. In addition $(l=2)$, we implement second level interventions with protective measures that increase the average effective contact reduction to $70 \%$ in total $(l=\{1,2\})$. For incidences above $100, l=1$ leads to $58 \%$ and $l=\{1,2\}$ to $76 \%$ of effective contact reduction. For the less stricter set denoted by $\mathbf{L}$, we have effective contact reductions $(l=\{1,2\})$ of $57 \%$ and $64 \%$ with incidences below and above 100, respectively. For the Monte Carlo runs, we vary the contact reduction in a range of $\pm 5 \%$.

The particular contact reduction values for red and green zones and different locations (homes, schools, workplaces, and other) are based on [11] and on model calibration: red zones must substantially reduce their incidences in lockdown over multiple weeks and green zones are calibrated to maintain stable low incidence values within the local population (i.e. if there weren't any commuters).

The second dimension of our strategy space is given by four different rates of commuter testing. With T0, there is no particular commuter testing at all, and with T1, T2, T5 we refer to testing rates of once, twice, and five times a week. Based on a 5 days working week, T5 will also be called the daily testing strategy. We assume massive deployment of antigen tests combined with a smaller number of PCR or RT-qPCR and pool tests [25, 26, 27] to control the commuter spread from highly infected regions. In [20], the sensitivity of antigen tests on the German market is estimated by $40-80 \%$, and [28] identifies average chances of $72 \%$ and $58 \%$ to correctly detect an infection by antigen tests for symptomatic and asymptomatic 
Lockdown strictness

1.) $\mathbf{L}+$ : effective contact reduction: $70 \%(I \leq 100), 76 \%(I>100)$

2.) L: effective contact reduction: $57 \%(I \leq 100), 64 \%(I>100)$

Commuter Testing

1.) T0: no testing

2.) $\mathrm{T} 1: 1$ test per week

3.) T2: 2 tests per week

4.) T5: 5 tests per week
Delay of implementation

1.) D1W: 1 week delay

2.) D3W: 3 weeks delay

Table 2 Tensor space of mitigation strategies. The 16 considered strategies are defined by choosing one item per column.

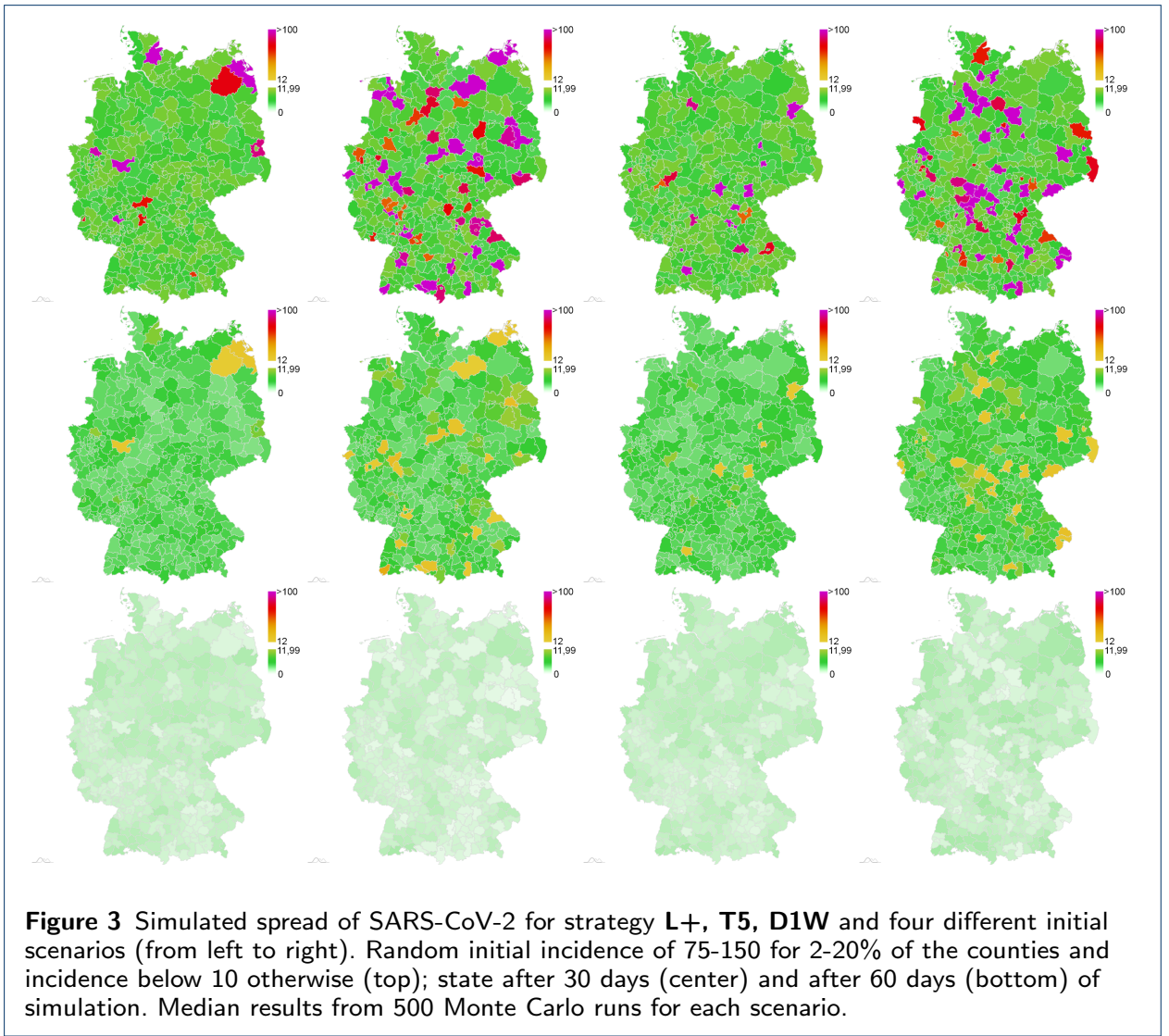

cases, respectively. Given a combination of different kinds of tests, we use a generic daily detection ratio of $75 \%$ for carriers and infectious persons.

The last key property in our strategy space is the delay of implementation of new interventions once critical thresholds are exceeded. We consider two different delays, a delay of one week denoted by $\mathbf{D} \mathbf{1 W}$ and a delay of three weeks denoted by D3W.

\section{Results}

In this section, we present numerical results for the simulation of the 16 defined strategies for 25 randomized initial scenarios. In particular, we focus on the most effective strategy consisting of strict lockdowns, daily testing, and fast implementation of interventions $(\mathbf{L}+, \mathbf{T} 5, \mathbf{D} 1 \mathbf{W})$.

In Fig. 3, we present four different scenarios of virus spread across Germany with 2 to $20 \%$ of the regions classified as red zones (incidence 75-150 at start). These are generic scenarios of local outbreaks as they have been observed over the last year [3] 


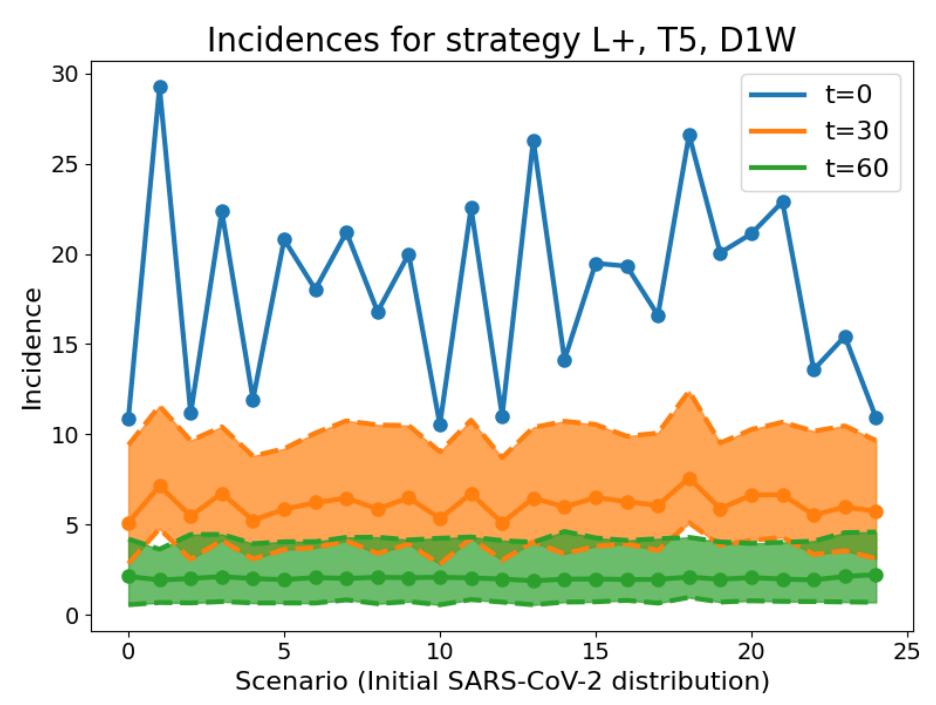

Figure 4 Country-wide SARS-CoV-2 infections per 100000 and seven days (denoted: incidence) with the strongest mitigation strategy after 30 days (orange) and after 60 days (green) with the initial setting shown by the blue curve. The p25 and p75 percentiles for 500 Monte Carlo runs and 30 and 60 days of simulation, respectively, are shown by dashed lines in the same color.

in a smaller quantity. Each red zone represents a snapshot of the situation before the virus spreads into the surrounding regions. The initial situation is depicted on the top. In the center, we see the median outcome after 30 days and on the bottom the outcome after 60 days. Already after thirty days, we have a stable incidence of about 5 and only a small number of counties with incidences around 20 or 30 . After 60 days, the initial heterogeneous situation is completely under control with stable incidences around 1 or 2 for all German counties.

In Fig. 4, we present the average incidence over the whole country for the 25 different initial red zone distributions. The median incidences (as well as the percentiles p25 and p75 of the Monte Carlo runs) for strategy L+,T5,D1W after 30 and 60 days are shown in orange and green, respectively. Analogous to the four scenarios considered in Fig. 3, the overall incidence drops considerably after 30 days already and is below 5 after 60 days in all 25 scenarios. As expected, a strong local lockdown $\mathbf{L}+$, daily testing and a fast response time lead to green zones remaining green and red zones becoming green after only 1-2 months and thus avoiding a perpetuation of interventions.

In Fig. 5 and Fig. 6, we present the results for all strategies and all 25 initial scenarios. In Fig. 5, we see the number of counties in lockdown for all mitigation strategies (averaged over the 25 different scenarios) for 30 days of simulation time. Note that we use the same color for strategies that only differ in the strength of the lockdown $\mathbf{L}+$ (solid) vs $\mathbf{L}$ (dashed). In all cases, the $\mathbf{L}+$ strategy leads to less counties in lockdown over time. Hence, the most important factor for controlling the dynamics is the strictness of the lockdown in the red zones. The reduced lockdown strength $\mathbf{L}$ with an effective contact reduction of $57 \%(I \leq 100)$ and $64 \%(I>100)$ needs to be complemented by daily testing $\mathbf{T} 5$ to control the propagation; see $\mathbf{L}$, T5, D1W and $\mathbf{L}, \mathbf{T 5}$, D3W. With $\mathbf{L}$ and lesser testing, the number of counties 


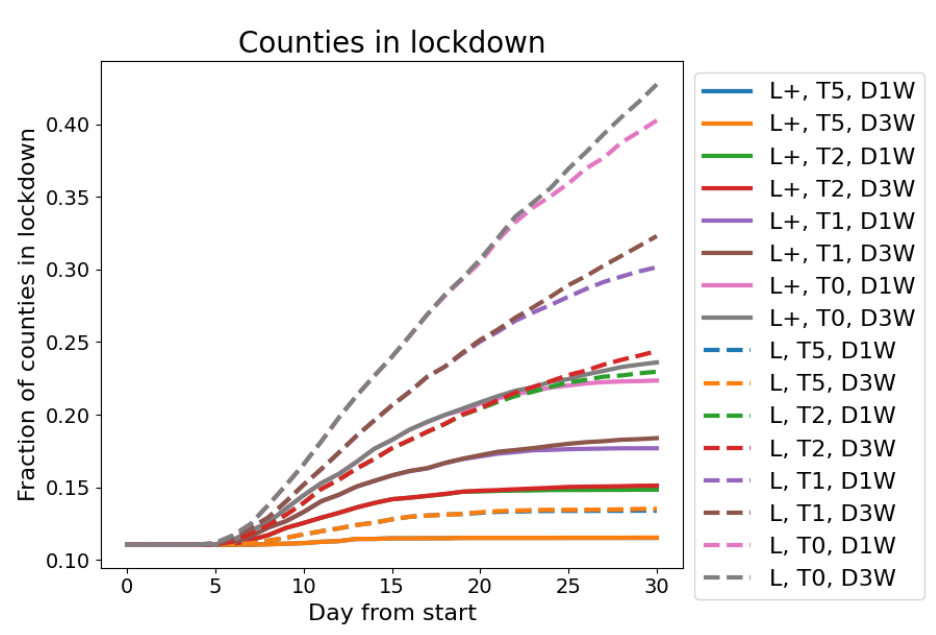

Figure 5 Counties in lockdown per day from start for the different mitigation strategies. Due to the strictness of the interventions in L+, T5, D1W and L+, T5, D3W, the delay of implementation is of minor importance and the curves overlap. For less strict interventions longer delays lead to more severe situations.

in lockdown doubles within just thirty days. Considering the slope of the curves, we see that the weaker the strictness of the interventions, the faster immediate action is required. With testing only once a week $(\mathbf{L}, \mathbf{T} \mathbf{1}, \mathbf{D} * \mathbf{W}, * \in 1,3)$, almost a third of all counties will be in lockdown after only one month. In order to control the dynamics with testing rates under twice a week, very strict lockdowns $\mathbf{L}+$ with effective contact reductions of $70 \%(I \leq 100)$ and $76 \%(I>100)$ have to be implemented. These would have to be stricter than anything decreed for Germany ever before.

Fig. 6 depicts how many lockdowns have to be implemented for each scenario and each strategy over the whole country, i.e., how many counties turn red that were green in the beginning. Lesser restrictions like lockdown $\mathbf{L}$ and testing T0, T1 or even T2 lead to a substantial number of necessary lockdowns in neighboring regions. This number is $20-30$ or even 100 times larger than that of strategy $\mathbf{L}+$, T5, D1W. Stronger containment measures lead to more opening possibilities and hence to less economical damage than a perpetuation of less effective measures as also established in [13, 29].

In Fig. 7, we depict the outcomes for one particular scenario of about $18 \%$ red zones after 30 days of simulation with all 16 opening and mitigation strategies presented in Table Table 2. Again, strategies $\mathbf{L}+, \mathbf{T 5}, \mathbf{D} \mathbf{1 W}$ and $\mathbf{L}+, \mathbf{T 5}, \mathbf{D} 3 \mathbf{W}$ lead to the most promising results. The images also quantify how reduced testing for the same lockdown strictness leads to slightly worse outcomes while reduced testing combined with reduced lockdown strictness quickly lead to out-of-control virus dynamics.

Finally, in Fig. 8, we present the outcome of the strongest and intermediate interventions, i.e. $\mathbf{L}+, \mathbf{T} 5, \mathbf{D} \mathbf{1} \mathbf{W}$ and $\mathbf{L}, \mathbf{T} \mathbf{2}, \mathbf{D} \mathbf{3} \mathbf{W}$, for the case of a much severe initial situation with $42 \%$ and $60 \%$ counties as red zones. After 30 days with $\mathbf{L}+$, T5, D1W, the situations improves considerably (center), while after thirty days with $\mathbf{L}, \mathbf{T} 2$, D3W, we see a deterioration of infection dynamics (right). 


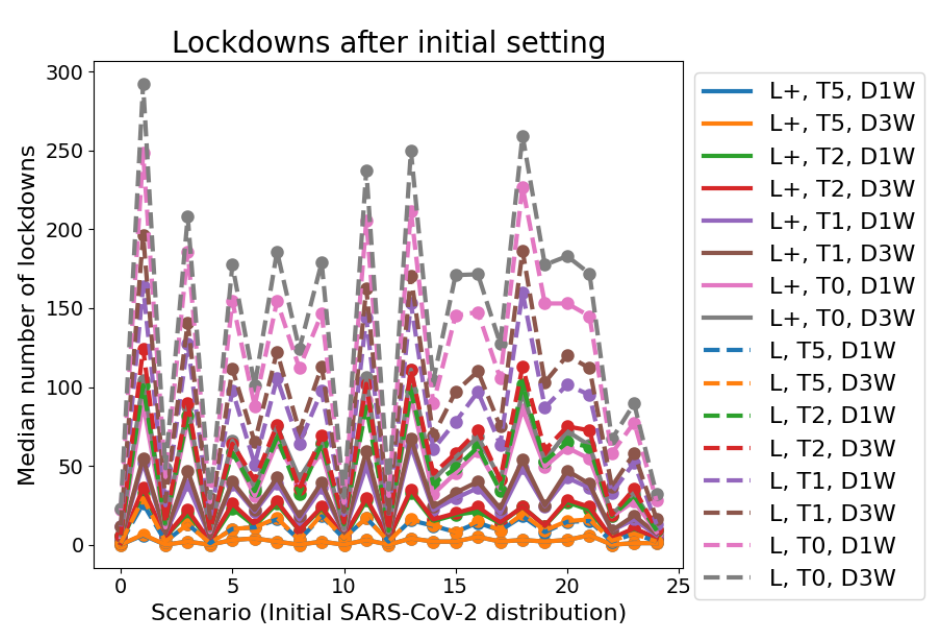

Figure 6 Median number of lockdowns per scenario (different initial distributions of SARS-CoV-2 spread) for the provided mitigation and opening strategies.

\section{Discussion}

Within the last year, a variety of different approaches to controlling the spread of the SARS-CoV-2 pandemic has been proposed. A key component in many successful interventions is a more or less differentiated and explicit zone distinction strategy based on regionally heterogeneous pandemic developments; see also Refs. [14, 15, 16]. Here, we investigated a basic version with only two different zones that are distinguished simply by incidence levels. While such zone distinction concepts are straightforward to implement in sparsely inhabited countries or countries with clear regional separation that facilitates isolation of high-incidence regions, the densely populated, highly mobile, highly connected situation in Germany, the EU, and similar regions presents challenges.

In particular, concepts of dealing with commuters between regions are needed. Work-related commuting between regions is often critical to maintaining a basic level of economic and social activity. Given the enormous cost of a complete ban on travel to neighboring cities, restricting mobility beyond a certain level seems unrealistic from a pragmatic point of view.

On the other hand, unrestricted travel between high- and low-incidence regions is problematic, as it effectively prevents regionally differentiated approaches due to the expected high amount of imported cases that will quickly revert any local progress towards lower incidence. Besides reducing unnecessary mobility between zones with different status to a minimum, testing commuters has been proposed as a tool to reduce import of cases into low-incidence zones. While this is intuitively appealing, its utility and practicality in a real-world scenario remains yet to be demonstrated.

Our modeling approach aims at providing a data-based estimate of the effect that a combination of regionally differentiated restrictions and systematic testing of commuters with a given test sensitivity and frequency has on the overall incidence, on the frequency of necessary lockdowns, and on the containment of isolated local outbreaks. A limitation of the approach is that border regions are not considered, e.g., the impact of systematically higher incidences in a neighbouring country. However, 
since our simulation approach consists of randomly initialized generic scenarios, our conclusions also hold true for mobility across German borders.

Our results clearly indicate that a combined strategy of local lockdowns and systematic testing has the potential to contain isolated local outbreaks in a general low incidence setting. In particular, rather strict local measures in combination with frequent testing of commuters proves highly effective in preventing the spread of localized infection hot spots and reducing the incidence in these regions. This can be seen both in the number of counties that need to impose measures and in the median incidence in the simulations which can be brought to surprisingly low levels with the most effective strategies.

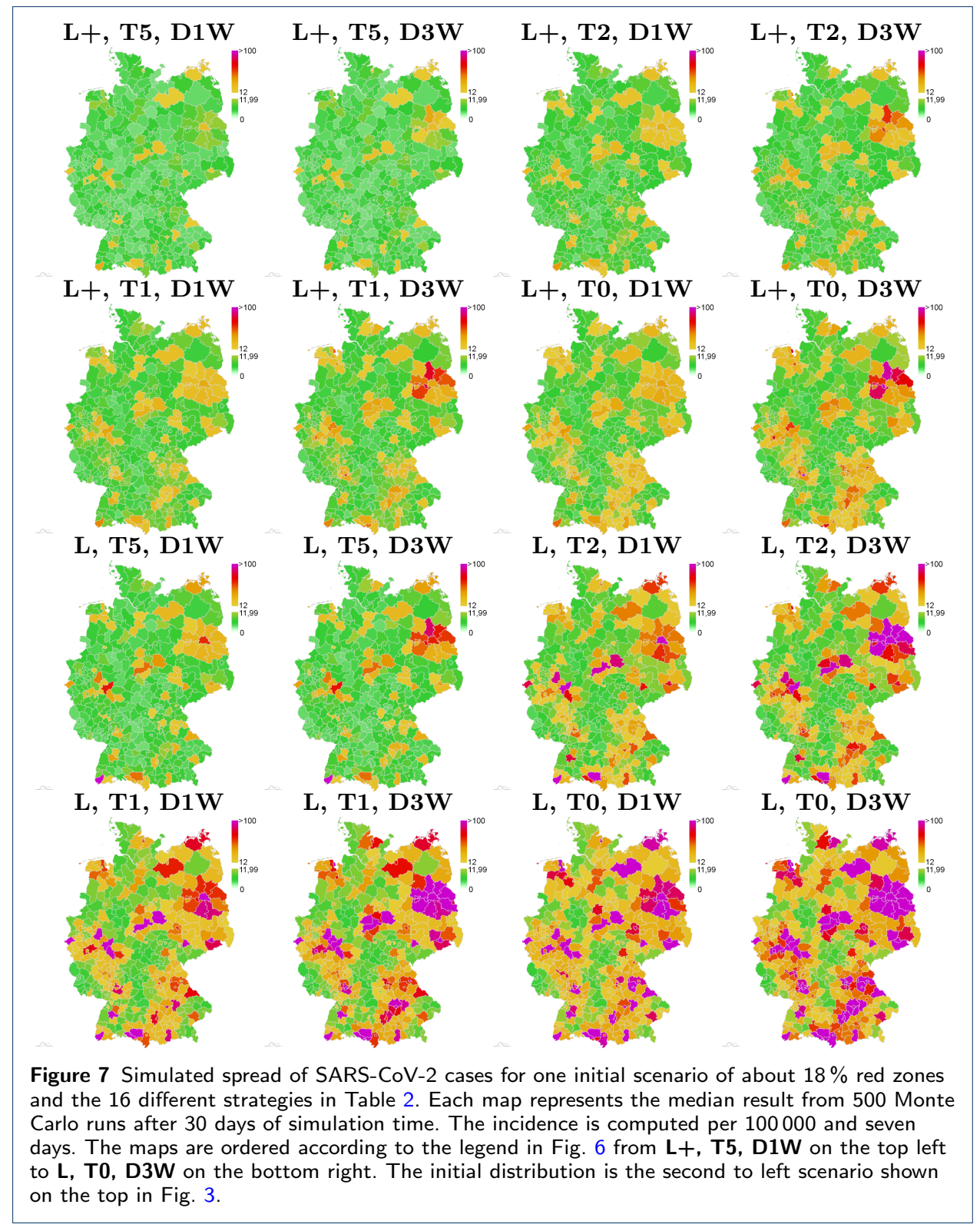

The results further clearly indicate a hierarchy of effectiveness that depends on the strength of the locally imposed measures, the duration of the delay with which 


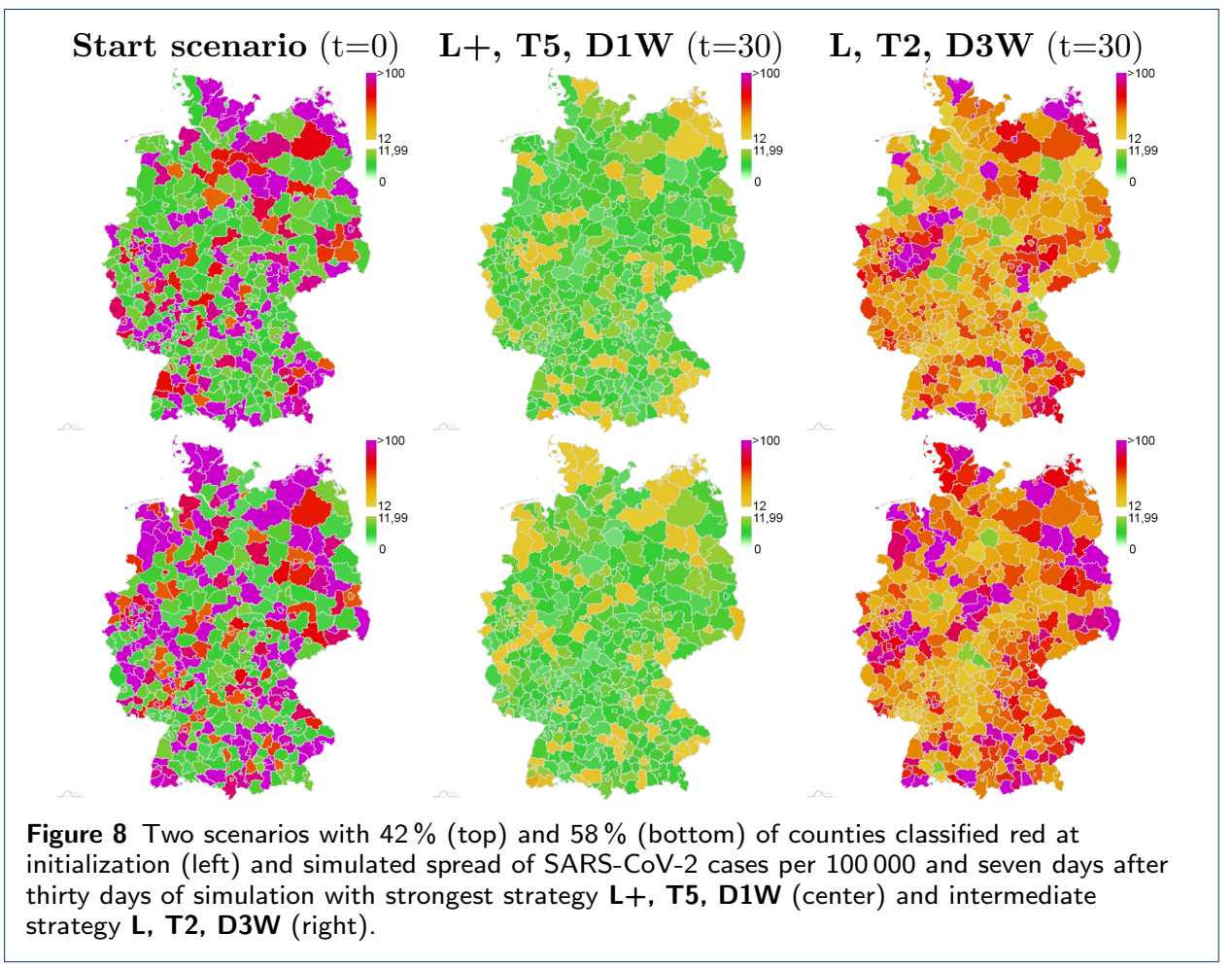

they are imposed after a critical incidence threshold has been reached, and the frequency at which commuters are tested which ultimately determines the fraction of imported cases that are found.

Interestingly, although the strength of the locally imposed measures dominates the further development of a local outbreak as expected, our simulations indicate that with an effective daily testing regimen and a swift reaction to local outbreaks, even a less severe reaction may be effective in containing isolated outbreaks. With less strict contact reduction policies in the lockdown scenario, our model predicts especially testing frequency to be a highly relevant factor in outbreak mitigation. It should be noted, however, that our scenarios are low-incidence scenarios with a rather small number of local infection hot spots. With a high overall incidence as demonstrated in Fig. 8, strict measures are even more essential in first instance. With very strict policy interventions, reaction delay and testing of commuters appear to have less of an impact. However, testing only once a week or not at all can quickly lead to a degeneration of infection dynamics.

\section{Conclusions}

Using a regionally resolved model based on mobility data to describe pandemic spread on a subnational level, we systematically investigated the feasibility of a localized strategy for outbreak containment based on a simple distinction of lowand high-incidence regions. Such a strategy may be especially useful in a scenario where generalized, nation-wide measures have brought incidence to a rather low overall level and a restrictive overall policy is no longer necessary. In that sense, it can be seen as a perspective for careful resumption of economic and social activity. This is especially important as there is a clear general pattern of re-emergence of 
the pandemic after successful containment and lifting of restrictive policy; e.g., the incidence in Germany remained at very low levels after the first outbreak in March 2020 until September when several local outbreaks emerged and, after lack of a substantial intervention, developed into a nationwide second surge of infections.

Our results indicate that local containment of outbreaks and maintenance of low overall incidence is possible even in densely populated and highly connected areas. While we demonstrate this on data from Germany, similar patterns of mobility likely exist in many countries and our results are, hence, generalizable to a certain extent.

While it is obvious that a substantial reduction of transmission and, hence, contacts in the population is necessary to mitigate a local high-incidence situation, our results suggest that reduced mobility along with frequent testing of regular commuters can successfully prevent generalized spreading of the infection in larger areas even with less strict contact reduction policies, especially when reaction to an outbreak is swift. This gives rise to a promising perspective after hard and economically damaging policy interventions: Maintaining the situation at stable levels may require moderate, localized interventions that affect only a small fraction of the population, offering a viable alternative to switching back and forth between restrictive untargeted measures and premature lifting of restrictions.

\title{
Declarations
}

\author{
Abbreviations \\ Covid-19:Coronavirus disease 2019, SARS-CoV-2: severe acute respiratory syndrome coronavirus type 2.
}

Ethics approval and consent to participate

Not applicable.

Consent for publication

Not applicable.

Availability of data and materials

The parameters to setup the simulations are provided in the current article and [11]. The simulation output for all different strategies, scenarios and Monte Carlo runs presented here make up more than $100 \mathrm{~GB}$ in over 1.9 million items and cannot be shared easily. Particular results can be shared upon request. Please contact

Martin.Kuehn@DLR.de. Intra- and intercounty contact and raw, non-normalized mobility data for initialization are provided in text files.

Competing interests

The authors declare that they have no competing interests.

Funding

This work has received funding from the European Union's Horizon 2020 research and innovation programme under grant agreement No 101003480 and by the Initiative and Networking Fund of the Helmholtz Association. It was supported by German Federal Ministry of Education and Research for the project CoViDec (FKZ: 01KI20102). The funding bodies had no role in the design of the study, collection, analysis, and interpretation of the results, or writing the manuscript.

Acknowledgements

Not applicable.

Authors' contributions

M.M.-H., S.B., M.J.K. and M.K. developed the mitigation strategies, M.J.K., D.A., M.K., J.K., W.K. and M.S. developed and implemented the graph SIR-type model with commuter testing, M.J.K., K.R. and W.K. developed the data analysis tools, J.G. and L.S. developed the visualization tools, M.M.-H. and A.B. supervised the study.

Author details

${ }^{1}$ Institute for Software Technology, German Aerospace Center, Cologne, Germany. ${ }^{2}$ Department of Systems Immunology and Braunschweig Integrated Centre of Systems Biology (BRICS), Helmholtz Centre for Infection Research, Braunschweig, Germany. 
References

1. Robert Koch Institut: RKI Covid-19 Germany (2021).

https: //experience.arcgis.com/experience/478220a4c454480e823b17327b2bf1d4 Accessed 2021-04-13

2. Robert Koch Institute: Impfdashboard. Technical report, Robert Koch Institut (2021).

https: //impfdashboard.de/ Accessed 2021-04-20

3. Robert Koch Institute: Coronavirus disease 2019 - Daily Situation Report of the Robert Koch Institute. Technical report, Robert Koch Institut (2021).

https://www.rki.de/DE/Content/InfAZ/N/Neuartiges_Coronavirus/Situationsberichte/ Accessed 2020-04-13

4. Volz, E., Mishra, S., Chand, M., Barrett, J.C., Johnson, R., Geidelberg, L., Hinsley, W.R., Laydon, D.J., Dabrera, G., O'Toole, Á., Amato, R., Ragonnet-Cronin, M., Harrison, I., Jackson, B., Ariani, C.V., Boyd, O., Loman, N.J., McCrone, J.T., Gonçalves, S., Jorgensen, D., Myers, R., Hill, V., Jackson, D.K., Gaythorpe, K., Groves, N., Sillitoe, J., Kwiatkowski, D.P., Flaxman, S., Ratmann, O., Bhatt, S., Hopkins, S., Gandy, A., Rambaut, A., Ferguson, N.M.: Transmission of sars-cov-2 lineage b.1.1.7 in england: Insights from linking epidemiological and genetic data. medRxiv (2021). doi:10.1101/2020.12.30.20249034. https://www.medrxiv.org/content/early/2021/01/04/2020.12.30.20249034.1.full.pdf

5. Schlosser, F., Maier, B.F., Jack, O., Hinrichs, D., Zachariae, A., Brockmann, D.: Covid-19 lockdown induces disease-mitigating structural changes in mobility networks. Proceedings of the National Academy of Sciences 117(52), 32883-32890 (2020). doi:10.1073/pnas.2012326117. https://www.pnas.org/content/117/52/32883.full.pdf

6. Köhler, J., Schwenkel, L., Koch, A., Berberich, J., Pauli, P., Allgöwer, F.: Robust and optimal predictive control of the covid-19 outbreak. Annual Reviews in Control (2020). doi:10.1016/j.arcontrol.2020.11.002

7. Keimer, A., Pflug, L.: Modeling infectious diseases using integro-differential equations: Optimal control strategies for policy decisions and Applications in COVID-19. Technical report, Friedrich-Alexander-Universität Erlangen-Nr̈nberg (2020). doi:10.13140/RG.2.2.10845.44000

8. Khailaie, S., Mitra, T., Bandyopadhyay, A., Schips, M., Mascheroni, P., Vanella, P., Lange, B., Binder, S.C., Meyer-Hermann, M.: Development of the reproduction number from coronavirus sars-cov-2 case data in germany and implications for political measures. BMC medicine 19(1), 1-16 (2021)

9. Kergaßner, A., Burkhardt, C., Lippold, D., Nistler, S., Kergaßner, M., Steinmann, P., Budday, D., Budday, S.: Meso-scale modeling of covid-19 spatio-temporal outbreak dynamics in germany. medRxiv (2020). doi:10.1101/2020.06.10.20126771

10. Barbarossa, M.V., Fuhrmann, J., Meinke, J.H., Krieg, S., Varma, H.V., Castelletti, N., Lippert, T.: Modeling the spread of covid-19 in germany: Early assessment and possible scenarios. PLOS ONE 15(9), 1-22 (2020). doi:10.1371/journal.pone.0238559

11. Kühn, M.J., Abele, D., Mitra, T., Koslow, W., Abedi, M., Rack, K., Siggel, M., Khailaie, S., Klitz, M., Binder, S., Spataro, L., Gilg, J., Kleinert, J., Häberle, M., Plötzke, L., Spinner, C.D., Stecher, M., Zhu, X.X., Basermann, A., Meyer-Hermann, M.: Assessment of effective mitigation and prediction of the spread of SARS-CoV-2 in Germany using demographic information and spatial resolution. medRxiv (2020). doi:10.1101/2020.12.18.20248509. https://www.medrxiv.org/content/early/2020/12/22/2020.12.18.20248509.full.pdf

12. Müller, S.A., Balmer, M., Charlton, W., Ewert, R., Neumann, A., Rakow, C., Schlenther, T., Nagel, K.: Predicting the effects of covid-19 related interventions in urban settings by combining activity-based modelling, agent-based simulation, and mobile phone data. medRxiv (2021). doi:10.1101/2021.02.27.21252583

13. Baumann, M., Beier, M., Brinkmann, M., Bude, H., Fuest, C., Feldner, D., Hallek, M., Kickbusch, I., Mayer, M., Meyer-Hermann, M., Peichl, A., Rosert, E., Schneider, M.: A proactive approach to fight sars-cov-2 in germany and europe. Technical report (2021). No-COVID: Controlling the Covid-19 pandemic through Green Zones. https://nocovid-europe.eu/assets/doc/nocovid_framework.pdf

14. Horton, R.: Offline: The case for no-covid. Lancet (London, England) 397(10272), 359 (2021)

15. Shen, C., Killeen, G.F., Staines, A., Bar-Yam, Y.: A green zone strategy for ireland. Technical report, New England Complex Systems Institute (2020). https://necsi.edu/a-green-zone-strategy-for-ireland

16. Jasanoff, S., Hilgartner, S., Hurlbut, J.B., Özgöde, O., Rayzberg, M.: Comparative covid response: Crisis, knowledge, politics. Technical report, Harvard Kennedy School (2021). https://necsi.edu/a-green-zone-strategy-for-ireland

17. Poletti, P., Tirani, M., Cereda, D., Trentini, F., Guzzetta, G., Marziano, V., Buoro, S., Riboli, S., Crottogini, L., Piccarreta, R., Piatti, A., Grasselli, G., Melegaro, A., Gramegna, M., Ajelli, M., Merler, S.: Age-specific SARS-CoV-2 infection fatality ratio and associated risk factors, Italy, February to April 2020. Eurosurveillance 25(31), 2001383 (2020). doi:10.2807/1560-7917.ES.2020.25.31.2001383

18. Levin, A.T., Hanage, W.P., Owusu-Boaitey, N., Cochran, K.B., Walsh, S.P., Meyerowitz-Katz, G.: Assessing the age specificity of infection fatality rates for Covid-19: systematic review, meta-analysis, and public policy implications. medRxiv, 2020-072320160895 (2020). doi:10.1101/2020.07.23.20160895

19. Verity, R., Okell, L.C., Dorigatti, I., Winskill, P., Whittaker, C., Imai, N., Cuomo-Dannenburg, G., Thompson, H., Walker, P.G.T., Fu, H., Dighe, A., Griffin, J.T., Baguelin, M., Bhatia, S., Boonyasiri, A., Cori, A., Cucunubá, Z., FitzJohn, R., Gaythorpe, K., Green, W., Hamlet, A., Hinsley, W., Laydon, D., Nedjati-Gilani, G., Riley, S., Elsland, S.v., Volz, E., Wang, H., Wang, Y., Xi, X., Donnelly, C.A., Ghani, A.C., Ferguson, N.M.: Estimates of the severity of coronavirus disease 2019: a model-based analysis. The Lancet Infectious Diseases 20(6), 669-677 (2020). doi:10.1016/S1473-3099(20)30243-7

20. Robert Koch Institute: Antigentests als ergänzendes Instrument in der Pandemiebekämpfung. Technical report, Robert Koch Institut (2021). https:

//www.rki.de/DE/Content/Infekt/EpidBull/Archiv/2021/Ausgaben/17_21.pdf?__blob=publicationFile Accessed 2021-04-19

21. BMAS: Pendlerverflechtungen der sozialversicherungspflichtig Beschäftigten nach Kreisen - Deutschland 
(Jahreszahlen) (2019). https://statistik.arbeitsagentur.de/SiteGlobals/Forms/Suche/ Einzelheftsuche_Formular.html?nn=20934\&topic_f=beschaeftigung-sozbe-krpend Accessed 2020-10-12 22. Twitter: Twitter Docs (2020). https://developer.twitter.com/

23. Prem, K., Cook, A.R., Jit, M.: Projecting social contact matrices in 152 countries using contact surveys and demographic data. PLoS computational biology 13(9), 1005697 (2017). doi:10.1371/journal.pcbi.1005697

24. Fumanelli, L., Ajelli, M., Manfredi, P., Vespignani, A., Merler, S.: Inferring the structure of social contacts from demographic data in the analysis of infectious diseases spread. PLoS Computational Biology 8(9), 1002673 (2012). doi:10.1371/journal.pcbi.1002673

25. Donoho, D., Lotfi, M., Ozturkler, B.: The Mathematics of Mass Testing for COVID-19. https://sinews.siam.org/Details-Page/the-mathematics-of-mass-testing-for-covid-19

26. Cherif, A., Grobe, N., Wang, X., Kotanko, P.: Simulation of Pool Testing to Identify Patients With Coronavirus Disease 2019 Under Conditions of Limited Test Availability. JAMA Network Open 3(6), 2013075-2013075 (2020). doi:10.1001/jamanetworkopen.2020.13075.

https://jamanetwork.com/journals/jamanetworkopen/articlepdf/2767513/cherif_2020_ld_200088.pdf

27. Sunjaya, A.F., Sunjaya, A.P.: Pooled testing for expanding covid-19 mass surveillance. Disaster Medicine and Public Health Preparedness 14(3), 42-43 (2020)

28. Dinnes, J., Deeks, J., Berhane, S., Taylor, M., Adriano, A., Davenport, C., Dittrich, S., Emperador, D., Takwoingi, Y., Cunningham, J., Beese, S., Domen, J., Dretzke, J., Ferrante di Ruffano, L., Harris, I., Price, M. Taylor-Phillips, S., Hooft, L., Leeflang, M., Mclnnes, M., Spijker, R., Van den Bruel, A.: Rapid, point?of?care antigen and molecular?based tests for diagnosis of SARS?CoV?2 infection. Cochrane Database of Systematic Reviews (3) (2021). doi:10.1002/14651858.CD013705.pub2

29. Dorn, F., Khailaie, S., Stöckli, M., Binder, S., Lange, B., Vanella, P., Wollmershäuser, T., Peichl, A., Fuest, C. Meyer-Hermann, M.: Das gemeinsame Interesse von Gesundheit und Wirtschaft: Eine Szenarienrechnung zur Eindämmung der Corona- Pandemie. Technical Report 6 (2020). https://www.ifo.de/publikationen/2020/ article-journal/das-gemeinsame-interesse-von-gesundheit-und-wirtschaft

\section{Additional Files}

Contact and mobility raw data - raw_mobility_contact.zip

This files includes the baseline intra-county contact patterns and the raw, non-normalized mobility data, not yet differentiated for age-groups, used for initialization of inter-county mobility. 